\title{
Cálculo da Probabilidade de Erro de Bit dos Sistemas M-QAM Quadrado e M-PAM em Desvanecimento Nakagami
}

\author{
Wamberto J. L. Queiroz, Waslon T. A. Lopes, Francisco Madeiro e Marcelo S. Alencar
}

\begin{abstract}
Resumo-Este trabalho apresenta expressões exatas para o cálculo da probabilidade de erro de bit de esquemas de modulação M-QAM e M-PAM, sob desvanecimento Nakagami, com base na consistência de mapeamento de constelações MQAM e M-PAM sob mapeamento de Gray.
\end{abstract}

Palavras-Chave-Desvanecimento Nakagami, modulação, mapeamento de Gray.

Abstract-This work presents exact expressions for calculating the bit error probability of M-QAM and M-PAM modulation schemes, under Nakagami fading, regarding the bit mapping consistency of M-QAM and M-PAM constelations with Gray mapping.

Keywords - Nakagami fading, modulation, Gray mapping.

\section{INTRODUÇÃO}

$\mathrm{O}$ uso de esquemas de modulação M-QAM (M-ary Quadrature Amplitude Modulation) em sistemas de comunicações modernos é consolidado e ocorre em vários tipos de modens síncronos de altas taxas de dados. Alguns exemplos são os modens 209A, da AT\&T, que transmitem dados síncronos com taxa $9600 \mathrm{~b} / \mathrm{s}$ em modo full-duplex usando modulação 16QAM, e o modelo MRD-5102 da Rockwell-Collins, que usa modulação 256-QAM. Outra aplicação são enlaces de fibras óticas para sistemas de TV a cabo de primeira geração - CATV (Cable TV). Os modens que são usados nesses sistemas para fornecer dados por cabo têm uma camada de rede IP (Internet Protocol) e a camada física de descida inclui os formatos de modulação 64-QAM e 256-QAM e códigos de correção de erro Reed-Solomon e de treliça. Na direção de subida, a camada física de transmissão do modem inclui os formatos de modulação QPSK (Quadrature Phase Shift Keying) e 16QAM.

$\mathrm{O}$ uso desse esquema de modulação em sistemas de transmissão sem fio se faz, ainda, nos padrões DVB-T (Digital Video Broadcasting - Terrestrial) e ISDB-T (Integrated Services Digital Broadcasting - Terrestrial) de TV digital, comercializados respectivamente no Reino Unido e no Japão a partir 1998. No padrão ISDB-T os dados de entrada do codificador MPEG-2 (Moving Picture Experts Group - 2) são

Wamberto José Lira Queiroz, Universidade Federal do Ceará, Sobral, Brasil, e-mail: wamberto@ufc.br. Waslon Terllizzie Araújo Lopes, ÁREA1 Faculdade de Ciência e Tecnologia, Salvador, Brasil, e-mail: waslon@area1.br. Francisco Madeiro, Universidade de Pernambuco, Recife, Brasil, e-mail: franciscomadeiro@yahoo.com.br. Marcelo Sampaio de Alencar, Universidade Federal de Campina Grande, Campina Grande, Brasil, e-mail: malencar@dee.ufcg.edu.br. Os autores também estão com o Instituto de Estudos Avançados em Comunicações(Iecom), Campina Grande, Brasil. arranjados em blocos de 188 bytes, chamados de MPEG2 TSP (Transport Stream Packet), enviados para um segmentador e em seguida são arranjados em um, dois ou três blocos de codificação. Esses blocos são chamados de camadas e seus dados passam por moduladores e podem ser mapeados em símbolos das constelações 16-QAM ou 64-QAM.

Esse processamento evita que o desvanecimento em rajadas prejudique as informações que trafegam pelo canal e tem recebido atenção de muitos pesquisadores [1]-[4]. Nesses trabalhos, os estudos têm sido desenvolvidos com o objetivo de avaliar o desempenho dos esquemas de modulação M-QAM usando como parâmetro de avaliação a probabilidade de erro de bit (PEB). Na referência [5], por exemplo, são mostradas aproximações para cálculo da PEB do esquema QAM obtidas a partir do uso de limitantes superiores ou inferiores, enquanto que em [1] é apresentada uma expressão mais apropriada para o cálculo da PEB sob ação do ruído AWGN (Additive White Gaussian Noise). Embora essas aproximações apresentem bons resultados para relações sinal ruído SNR (Signalto-Noise Ratio) elevadas, elas diferem dos valores exatos em SNRs baixas.

Uma contribuição a essa linha de estudos foi dada na referência [6], por Cho e Yoon, que apresentam expressões matemáticas exatas para cálculo da PEB de sistemas M-QAM e M-PAM sob ruído aditivo AWGN, usando mapeamento Gray. Em relação ao desvanecimento Rayleigh, podem ser citadas a referência [3], que fornece expressões para cálculo da PEB dos esquemas 16-QAM e 64-QAM em termos de uma integral definida da função $\mathrm{Q}(x)$, e a referência [4], em que os autores mostraram que um sinal recebido em um canal com desvanecimento Rayleigh é sujeito a uma distorção multiplicativa e a um ruído aditivo. Assim, após a compensação da distorção, o sinal na entrada do receptor pode ser visto como um sinal que apresenta um único termo de distorção aditiva que engloba os efeitos do ruído aditivo original, da distorção multplicativa e do erro de compensação dessa distorção.

Em [7] foi mostrado que o canal com desvanecimento Rayleigh pode ser visto como um canal sujeito a ruído aditivo. Esse ruído é modelado como a razão entre uma variável aleatória (v.a) gaussiana e uma v.a Rayleigh. A expressão da PEB para os esquemas M-QAM foi então obtida, usando o resultado apresentado em [6], substituindo a função $\operatorname{erfc}(x)$ pela função cumulativa de probabilidade (FCP) do novo ruído obtido. Essa mesma linha de raciocínio é usada nas próximas seções no cálculo das expressões da PEB para a constelação 
M-QAM quadrada e M-PAM sob ação do desvanecimento modelado pela distribuição de Nakagami.

\section{Formulação do Problema}

Em um ambiente de propagação com desvanecimento, a expressão matemática do sinal recebido na entrada do detector ótimo [5] é geralmente escrita como

$$
r_{c}(t)=\alpha e^{-j \phi} s(t)+z(t), \quad 0 \leq t \leq T,
$$

em que $\alpha$ é uma variável aleatória que representa as variações de amplitude sofridas pelo sinal ao longo de seu percurso de propagação, $\phi$ é a variação de fase imposta pelo canal, $z(t)$ é um processo gaussiano aditivo branco de média nula e densidade espectral de potência (DEP) $N_{0} / 2$ e $T$ é a duração do símbolo transmitido.

Admitindo que o detector ótimo faça uma perfeita estimação das variações de fase $\phi$ sofridas pelo sinal transmitido, o sinal recebido na entrada do detector pode ser escrito, de acordo com [8], como

$$
r(t)=r_{c}(t) e^{j \phi}=\alpha s(t)+z(t) e^{j \phi}=\alpha s(t)+n(t) .
$$

Note, pela Equação (2), que a variável $n(t)$ continua sendo um processo de média nula e DEP $N_{0} / 2$.

No processo de detecção do sinal transmitido, baseado no critério da máxima probabilidade a posteriori, o detector ótimo pode utilizar duas estratégias diferentes para estimar o símbolo transmitido a partir da observação do sinal recebido $r(t)$. Uma estratégia é amplificar o sinal recebido para normalizar o ganho do canal de modo que as regiões de decisão do receptor correspondam às regiões de decisão da constelação dos sinais transmitidos e escolher como símbolo detectado aquele que minimiza a métrica $\left|r-\alpha s_{i}\right|$. A outra estratégia é usar o ganho do canal para amplificar a constelação dos sinais, comparar $r / \alpha$ com todos os símbolos transmitidos e escolher o símbolo que minimiza a métrica $\left|r / \alpha-s_{i}\right|$.

Em relação à modulação QPSK, foi mostrado na referência [7] que ambas as estratégias apresentam o mesmo desempenho em relação à probabilidade de erro de bit para um desvanecimento Rayleigh. Neste trabalho, a segunda estratégia é utilizada em um canal com desvanecimento modelado pela distribuição de Nakagami para obter as expresões da probabilidade de erro de bit para os esquemas de modulação M-PAM e M-QAM, tomando-se como base as expressões obtidas por Cho e Yoon em [6]. Para que se possa chegar ao resultado desejado é necessário, antes, obter a função cumulativa de probabilidade da nova variável aleatória definida como a razão entre uma variável aleatória gaussiana $N$ e uma variável aleatória $\alpha$ com distribuição de Nakagami, $L=N / \alpha$.

\section{Função Cumulativa de Probabilidade de $L=N / \alpha$}

A variável aleatória $N$ de média nula e variância $N_{0} / 2$, que modela o ruído gaussiano branco, tem sua função densidade de probabilidade (fdp) dada por

$$
p_{N}(n)=\frac{1}{\sqrt{\pi N_{0}}} e^{-n^{2} / N_{0}}
$$

enquanto que a distribuição de Nakagami da variável $\alpha$ é dada por

$$
p(\alpha)=\frac{2 m^{m} \alpha^{2 m-1}}{\Gamma(m) \Omega^{m}} e^{-\frac{m \alpha^{2}}{\Omega}} u(\alpha),
$$

em que $m$ é um parâmetro que controla a intensidade do desvanecimento, $\Omega$ é a potência média do envelope do sinal transmitido e $u(\cdot)$ é a função degrau unitário.

De acordo com [9], a função densidade de probabilidade de uma variável aleatória $L$, definida como a razão entre outras duas variáveis, $L=\frac{N}{\alpha}$, é dada por

$$
p_{L}(l)=\int_{-\infty}^{\infty}|\alpha| p(l \alpha, \alpha) d \alpha,
$$

em que a fdp conjunta $p(l \alpha, \alpha)$ é obtida substituindo-se o termo $n$ por $l \alpha$ na fdp

$$
p(n, \alpha)=\frac{2 m^{m} \alpha^{2 m-1}}{\Gamma(m) \Omega^{m}} e^{-\frac{m \alpha^{2}}{\Omega}} \frac{e^{-\frac{n^{2}}{N_{0}}}}{\sqrt{\pi N_{0}}} u(\alpha) .
$$

Como as variáveis aleatória $N$ e $\alpha$ são independentes, a fdp $p_{L}(l)$ é obtida integrando-se a fdp conjunta da Equação (6), obtendo-se

$$
\begin{aligned}
p_{L}(l) & =\int_{-\infty}^{\infty} \frac{|\alpha| 2 m^{m} \alpha^{2 m-1}}{\Gamma(m) \Omega^{m}} \frac{e^{-\frac{m \alpha^{2}}{\Omega}}}{\sqrt{\pi N_{0}}} e^{-\frac{l^{2} \alpha^{2}}{N_{0}}} u(\alpha) d \alpha \\
& =\frac{2}{\sqrt{\pi N_{0}}} \frac{m^{m}}{\Gamma(m)} \frac{1}{\Omega^{m}} \int_{0}^{\infty} \alpha^{2 m} e^{-\alpha^{2}\left(\frac{m}{\Omega}+\frac{l^{2}}{N_{0}}\right)} d \alpha .
\end{aligned}
$$

Nota-se pela Equação (7) que a última integral é da forma

$$
\int_{0}^{\infty} x^{2 m} e^{-\rho x^{2}} d x
$$

e seu resultado é obtido seguindo o desenvolvimento

$$
\begin{aligned}
\int_{0}^{\infty} x^{2 m} e^{-\rho x^{2}} d x & =\int_{0}^{\infty} x^{2 m} e^{-(\sqrt{\rho} x)^{2}} d x \\
& =\int_{0}^{\infty}\left(\frac{u}{\sqrt{\rho}}\right)^{2 m} e^{-u^{2}} \frac{d u}{\sqrt{\rho}} \\
& =\frac{1}{2 \rho^{\left(m+\frac{1}{2}\right)}} \Gamma\left(m+\frac{1}{2}\right) .
\end{aligned}
$$

Desse modo, tem-se que

$$
\begin{aligned}
\int_{0}^{\infty} \alpha^{2 m} e^{-\alpha^{2}\left(\frac{m}{\Omega}+\frac{l^{2}}{N_{0}}\right)} d \alpha= & \frac{1}{2} \Gamma\left(m+\frac{1}{2}\right) \times \\
& \left(\frac{l^{2}}{N_{0}}+\frac{m}{\Omega}\right)^{-\left(m+\frac{1}{2}\right)} .
\end{aligned}
$$

Substituindo esse resultado na Equação (7), obtém-se que a fdp da variável aleatória $L$, que representa o novo ruído aditivo, pode ser escrita como

$$
p_{L}(l)=\frac{1}{\sqrt{\pi N_{o}}} \frac{m^{m}}{\Omega^{m}} \frac{\Gamma\left(m+\frac{1}{2}\right)}{\Gamma(m)}\left(\frac{l^{2}}{N_{o}}+\frac{m}{\Omega}\right)^{-\left(m+\frac{1}{2}\right)},
$$

que ainda pode ser escrita como

$$
p_{L}(l)=\frac{m^{m}}{\sqrt{\pi} \Omega^{m}} \frac{\Gamma\left(m+\frac{1}{2}\right)}{\sqrt{N_{o}} \Gamma(m)}\left(\frac{l^{2} \Omega+m N_{o}}{N_{o} \Omega}\right)^{-m-\frac{1}{2}} .
$$


A FCP em função de $l, P_{L}(l)$, considerando-se $\Omega=1$ é então obtida calculando-se a integral

$$
\begin{aligned}
P_{L}(l)=\int_{-\infty}^{l} p_{L}(x) d x= & \frac{m^{m} N_{o}^{m}}{\sqrt{\pi}} \frac{\Gamma\left(m+\frac{1}{2}\right)}{\Gamma(m)} \times \\
& \int_{-\infty}^{l}\left(x^{2}+N_{o} m\right)^{-\frac{2 m+1}{2}} d x,
\end{aligned}
$$

em que

$$
\int\left(x^{2}+a\right)^{-\frac{2 m+1}{2}} d x=x_{2} F_{1}\left(\frac{1}{2}, m+\frac{1}{2} ; \frac{3}{2} ;-\frac{x^{2}}{a}\right) a^{-\left(m+\frac{1}{2}\right)}
$$

e ${ }_{2} F_{1}(a, b ; c ; x)$ é mais conhecida como função hipergeométrica de Gauss.

A avaliação desse resultado em $x$ tendendo para $-\infty$ é feita considerando a forma integral de ${ }_{2} F_{1}(a, b ; c ; x)$ [10] e tomando o limite

$$
\lim _{x \rightarrow-\infty} x_{2} F_{1}\left(\frac{1}{2}, m+\frac{1}{2} ; \frac{3}{2} ;-x^{2}\right) .
$$

O resultado dessa avaliação é a função $P_{L}(l)$ dada por

$$
\begin{aligned}
P_{L}(l)= & \frac{\Gamma\left(m+\frac{1}{2}\right)}{\sqrt{\pi} \Gamma(m)}\left\{\frac{l}{\sqrt{N_{o} m}}{ }_{2} F_{1}\left(\frac{1}{2}, m+\frac{1}{2} ; \frac{3}{2} ;-\frac{l^{2}}{N_{o} m}\right)\right. \\
& \left.+\frac{\Gamma\left(\frac{3}{2}\right) \mathrm{B}(m, 1-m)}{\Gamma\left(m+\frac{1}{2}\right) \Gamma(1-m)}\right\},
\end{aligned}
$$

em que $B(r, s)$ é a função beta de parâmetros $r$ e $s$.

\section{Probabilidade De ERro do Sistema M-QAM}

Essa FCP pode ser usada, por exemplo, no cálculo da propabilidade de erro de bit de um sistema M-QAM quadrado na presença de desvanecimento Nakagami, usando-se o resultado obtido no artigo [6], no qual os autores mostram que a PEB de um esquema M-QAM quadrado na presença de ruído gaussiano pode ser escrita como

$$
P_{b}=\frac{1}{\log _{2} \sqrt{M}} \sum_{k=1}^{\log _{2} \sqrt{M}} P_{b}(k)
$$

em que $P_{b}(k)$ é dado por

$$
\begin{array}{r}
P_{b}(k)=\frac{1}{\sqrt{M}} \sum_{i=0}^{\left(1-2^{-k}\right) \sqrt{M}-1}\{w(i, k, M) \times \\
\left.\operatorname{erfc}\left((2 i+1) \sqrt{\frac{3 \log _{2} M \gamma}{2(M-1)}}\right)\right\}
\end{array}
$$

e os pesos $w(i, k, M)$ são dados por

$$
w(i, k, M)=(-1)^{\left\lfloor\frac{i 2^{k-1}}{\sqrt{M}}\right\rfloor} \cdot\left(2^{K-1}-\left\lfloor\frac{i \cdot 2^{k-1}}{\sqrt{M}}+\frac{1}{2}\right\rfloor\right),
$$

com $\gamma=E_{b} / N_{0}$, energia de bit sobre a energia do ruído.

Sabendo então que $\operatorname{erfc}(x)=2 \mathrm{Q}(\sqrt{2} x)$ e que $\mathrm{Q}(x)$ é por definição a probabilidade de que uma variável aleatória gaussiana de média nula e variância unitária tenha valor maior que $x$, tem-se que

$$
\begin{aligned}
\operatorname{erfc} & \left(\sqrt{\frac{3(2 i+1)^{2} \log _{2}(M) E_{b}}{2(M-1)}}\right)= \\
= & Q\left(\sqrt{2} \sqrt{\frac{3(2 i+1)^{2} \log _{2}(M) E_{b}}{2(M-1)}}\right) \\
= & 2 \operatorname{Prob}\left(l \geq \sqrt{\frac{3(2 i+1)^{2} \log _{2}(M) E_{b}}{(M-1)}}\right) .
\end{aligned}
$$

A probabilidade de que a intensidade do novo ruído aditivo $l(t)$ exceda $\sqrt{\frac{3(2 i+1)^{2} \log _{2}(M) E_{b}}{(M-1)}}$ pode ainda ser escrita como

$$
\begin{aligned}
\operatorname{Prob} & \left(l \geq \sqrt{\frac{3(2 i+1)^{2} \log _{2}(M) E_{b}}{(M-1)}}\right)= \\
& =\int_{\sqrt{\frac{3(2 i+1)^{2} \log _{2}(M) E_{b}}{(M-1)}}}^{\infty} p_{L}(l) d l \\
& =1-P_{L}\left(\sqrt{\frac{3(2 i+1)^{2} \log _{2}(M) E_{b}}{(M-1)}}\right) .
\end{aligned}
$$

Aplicando esse resultado à FCP obtida na Equação (16) tem-se

$$
\begin{aligned}
P_{L} & \left(\sqrt{\frac{3(2 i+1)^{2} \log _{2}(M) E_{b}}{(M-1)}}\right)= \\
& =\frac{\Gamma\left(m+\frac{1}{2}\right)}{\sqrt{\pi} \Gamma(m)} \sqrt{\frac{3(2 i+1)^{2} \log _{2}(M)}{m(M-1)} \frac{E_{b}}{N_{0}}} \\
& \times{ }_{2} F_{1}\left(\frac{1}{2}, m+\frac{1}{2} ; \frac{3}{2} ;-\frac{3(2 i+1)^{2} \log _{2}(M)}{m(M-1)} \frac{E_{b}}{N_{0}}\right) \\
& +\frac{\mathrm{B}(m, 1-m)}{2 \Gamma(m) \Gamma(1-m)} .
\end{aligned}
$$

Desse modo,

$$
\begin{aligned}
2 \operatorname{Prob}\left(l \geq \sqrt{\frac{3(2 i+1)^{2} \log _{2}(M) E_{b}}{(M-1)}}\right)= \\
=2\left\{1-\frac{\Gamma\left(m+\frac{1}{2}\right)}{\Gamma(m) \sqrt{\pi}} \sqrt{\frac{3(2 i+1)^{2} \log _{2}(M)}{m(M-1)} \frac{E_{b}}{N_{0}}}\right. \\
\quad \times{ }_{2} F_{1}\left(\frac{1}{2}, m+\frac{1}{2} ; \frac{3}{2} ;-\frac{3(2 i+1)^{2} \log _{2}(M)}{m(M-1)} \frac{E_{b}}{N_{0}}\right) \\
\left.-\frac{\mathrm{B}(m, 1-m)}{2 \Gamma(m) \Gamma(1-m)}\right\} .
\end{aligned}
$$

A probabilidade de erro de bit do M-QAM quadrado pode 
ser escrita então como

$$
\begin{aligned}
P_{b} & =\frac{1}{\log _{2} \sqrt{M}} \sum_{k=1}^{\log _{2} \sqrt{M}} P_{b}(k) \\
P_{b}(k) & =\frac{2}{\sqrt{M}} \sum_{i=0}^{\left(1-2^{-k}\right) \sqrt{M}-1} w(i, k, M) \\
& \left\{1-\frac{\Gamma\left(m+\frac{1}{2}\right)}{\Gamma(m) \sqrt{\pi}} \sqrt{\frac{a_{i}(M) \gamma}{m}}\right. \\
& \left.\times{ }_{2} F_{1}\left(\frac{1}{2}, m+\frac{1}{2} ; \frac{3}{2} ;-\frac{a_{i}(M) \gamma}{m}\right)-\frac{\mathrm{B}(m, 1-m)}{2 \Gamma(m) \Gamma(1-m)}\right\},
\end{aligned}
$$

em que $a_{i}(M)=\frac{3(2 i+1)^{2} \log _{2}(M)}{M-1}$ e $\gamma=E_{b} / N_{o}$.

Uma expressão alternativa para o cálculo da PEB para valores inteiros de $m$ pode ser obtida fazendo-se a substituição de variáveis $x=\operatorname{tg}(\theta)$ na integral da Equação (14). O resultado dessa integral é dado por

$\int \frac{1}{\left(x^{2}+a\right)^{m+\frac{1}{2}}} d x=\frac{1}{a^{m}} \sum_{k=0}^{(m-1)} \frac{(-1)^{k} C_{m-1, k}}{(2 k+1)}\left[\frac{x}{\sqrt{x^{2}+a}}\right]^{2 k+1}$,

em que $C_{m-1, k}=\frac{(m-1) !}{k !(m-1-k) !}$.

Avaliando a Equação $(25) \mathrm{em}-\infty$ e $l$, a função $P_{L}(l)$ pode ser escrita como

$$
\begin{aligned}
P_{L}(l)=\frac{\Gamma\left(m+\frac{1}{2}\right)}{\sqrt{\pi} \Gamma(m)} & \sum_{k=0}^{(m-1)} \frac{(-1)^{k} C_{m-1, k}}{(2 k+1)} \times \\
& \left\{\left[\frac{l}{\sqrt{l^{2}+N_{0} m}}\right]^{2 k+1}+1\right\} .
\end{aligned}
$$

Substituindo esse resultado no lugar da função $\operatorname{erfc}(\cdot)$ na Equação (20) tem-se

$$
\begin{aligned}
P_{b}(k)= & \frac{2}{\sqrt{M}} \sum_{i=0}^{\left(1-2^{-k}\right) \sqrt{M}-1} w(i, k, M) \times \\
& \operatorname{Prob}\left(l \geq \sqrt{\frac{3(2 i+1)^{2}}{(M-1)} \log _{2}(M) E_{b}}\right) \\
= & \frac{2}{\sqrt{M}} \sum_{i=0}^{\left(1-2^{-k}\right) \sqrt{M}-1} w(i, k, M) \times \\
& \left\{1-\frac{\Gamma\left(m+\frac{1}{2}\right)}{\sqrt{\pi} \Gamma(m)} \sum_{k=0}^{(m-1)} \frac{(-1)^{k} C_{m-1, k}}{(2 k+1)}\right. \\
& \left.\times\left[\left(\frac{a_{i}(M) \gamma}{a_{i}(M) \gamma+m}\right)^{k+\frac{1}{2}}+1\right]\right\},
\end{aligned}
$$

de onde se obtém diretamente a probabilidade de erro $P_{b}$ [7].

\section{Probabilidade DE ERRO DO SISTEMA M-PAM}

A obtenção de uma expressão para a probabilidade de erro de bit do sistema M-PAM é feita usando o fato que a distância euclidiana entre os símbolos M-PAM é dada por

$$
d=\sqrt{\frac{3 \log _{2}(M) \cdot E_{b}}{\left(M^{2}-1\right)}}
$$

e que a expressão para o ruído gaussiano aditivo, $P_{b}$, de acordo com [6], é dada por

$$
P_{b}=\frac{1}{\log _{2}(M)} \sum_{k=1}^{\log _{2}(M)} P_{b}(k)
$$

com

$$
\begin{aligned}
P_{b}(k)=\frac{1}{M} \sum_{i=0}^{\left(1-2^{-k}\right) M-1}\{ & w(i, k, M) \times \\
& \left.\operatorname{erfc}\left((2 i+1) \sqrt{\frac{3 \log _{2} M \gamma}{\left(M^{2}-1\right)}}\right)\right\},
\end{aligned}
$$

$\mathrm{e}$

$$
w(i, k, M)=(-1)^{\left\lfloor\frac{i 2^{k-1}}{M}\right\rfloor} \cdot\left(2^{K-1}-\left\lfloor\frac{i \cdot 2^{k-1}}{M}+\frac{1}{2}\right\rfloor\right) .
$$

Seguindo então um procedimento semelhante ao usado na modulação M-QAM, encontra-se que

$$
\begin{aligned}
P_{b}(k) & =\frac{2}{M} \sum_{i=0}^{\left(1-2^{-k}\right) M-1} w(i, k, M) \\
& \left\{1-\frac{\Gamma\left(m+\frac{1}{2}\right)}{\Gamma(m) \sqrt{\pi}} \sqrt{\frac{a_{i}(M) \gamma}{m}}\right. \\
& \left.\times{ }_{2} F_{1}\left(\frac{1}{2}, m+\frac{1}{2} ; \frac{3}{2} ;-\frac{a_{i}(M) \gamma}{m}\right)-\frac{\mathrm{B}(m, 1-m)}{2 \Gamma(m) \Gamma(1-m)}\right\},
\end{aligned}
$$

em que $a_{i}(M)=\frac{6(2 i+1)^{2}}{\left(M^{2}-1\right)} \log _{2} M$ e $\gamma=E_{b} / N_{0}$.

É interessante observar neste ponto que as expressões obtidas nas seções anteriores, em termos das funções hipergeométrica, gamma e beta, convergem para o resultado obtido no artigo [7] quando $m=1$, para o desvanecimento Rayleigh. Conseqüentemente, os gráficos de probabilidade de erro de bits para o valor de $m=0.95$, próximo de $m=1$, como por exemplo na Figura 4, se aproximam dos gráficos obtidos para $m=1$ na Figura 6. Para $m=1$ e $M=16$, os resultados obtidos nesse artigo para o sistema $16-Q A M$ estão também compatíveis com os resultados obtidos por Shayesteh e Aghamohammadi em [4].

\section{RESULTADOS NUMÉRICOS}

As Figs. 1, 2 e 3 a seguir mostram as curvas de erro de bit traçadas a partir da Equação 24 para os esquemas de modulação 16-QAM, 64-QAM e 256-QAM para diferentes valores do parâmetro $m$ da distribuição de Nakagami. Percebese nos três casos que a probabilidade de erros aumenta quando $m$ diminui para 0.51. Quando $m=0.5$ a distribuição de Nakagami tende para a distribuição Gaussiana unilateral e quando $m$ aumenta para 0.95 a distribuição se aproxima da distribuição de Rayleigh. 


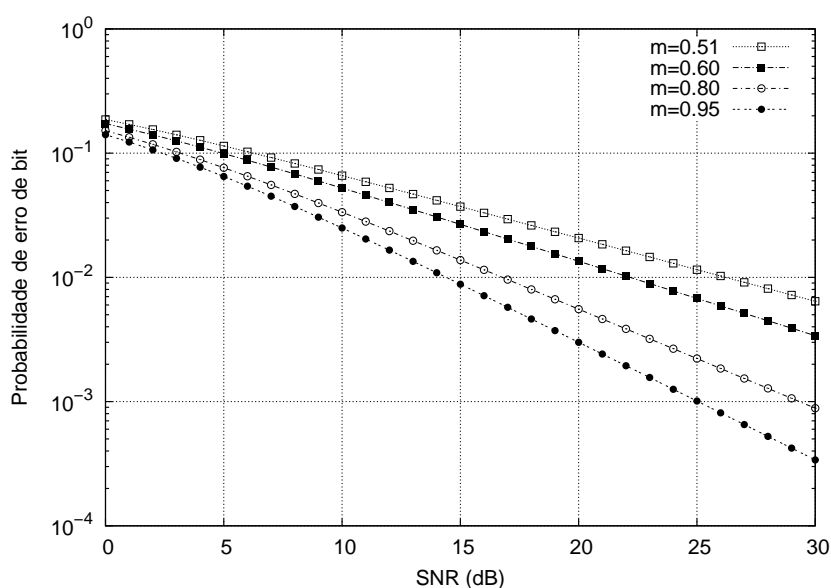

Fig. 1. Probabilidade de erro de bit de um sistema 16-QAM sob desvanecimento Nakagami para diferentes valores de $m, \frac{1}{2}<m<1$.

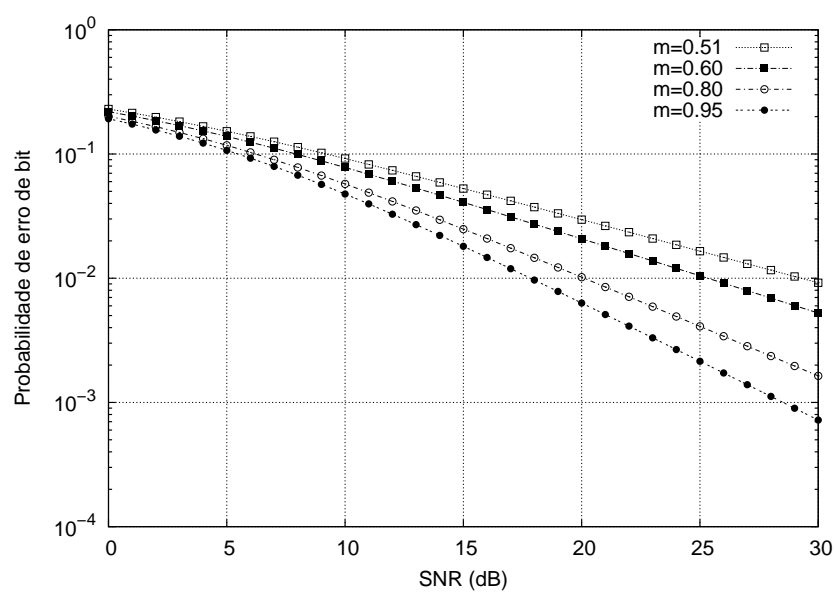

Fig. 2. Probabilidade de de erro de bit de um sistema 64-QAM sob desvanecimento Nakagami para diferentes valores de $m, \frac{1}{2}<m<1$.

Na Fig. 4 são mostradas curvas de probabilidade de erro de bit agrupadas para o mesmo valor de $m$ e para diferentes valores de $M$. Percebe-se pela Fig. 4 que o sistema 256QAM é mais susceptível ao desvanecimento que o sistema 16QAM. Isso ocorre porque, para uma mesma SNR, a distância euclidiana entre os componentes de dois símbolos distintos da constelação 256-QAM é menor do que na constelação 16QAM.

Na Fig. 5 são mostradas as curvas de probabilidade de erro de bit para o esquema 256-QAM para diferentes valores de $m$ maiores que 1 . À medida que $m$ aumenta, a intensidade do desvanecimento diminui e o esquema de modulação sofre menos erros.

Um outro resultado interessante é o comportamento da probabilidade de erro de bit para $m=1$, que corresponde ao desvanecimento Rayleigh. Quando $m=4$, por exemplo, tem-se a menor intensidade de desvanecimento entre os resultados numéricos apresentados. Esses resultados são mostrados respectivamente na Figs. 6 e 7.

Os resultados apresentados nas Figs. 6 e 7 mostram novamente que os esquemas com constelações maiores são mais

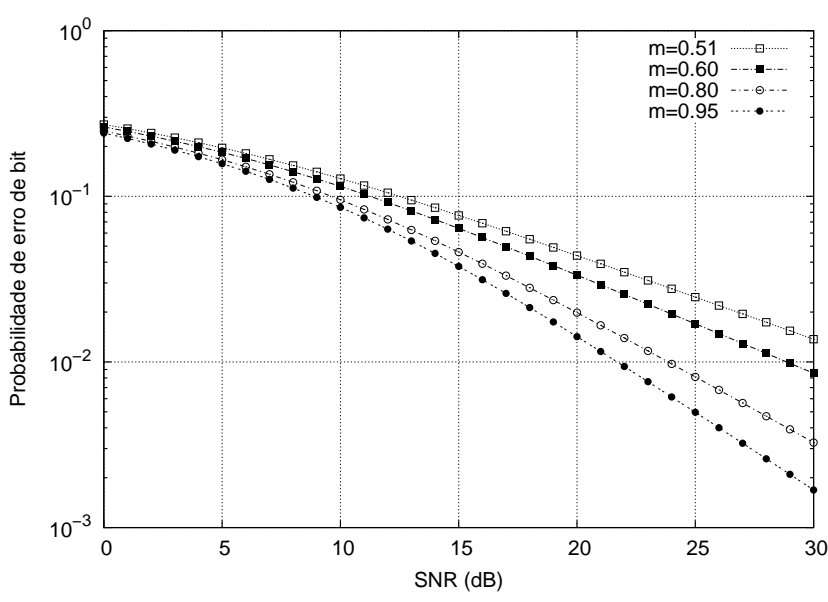

Fig. 3. Probabilidade de de erro de bit de um sistema 256-QAM sob desvanecimento Nakagami para diferentes valores de $m, \frac{1}{2}<m<1$.

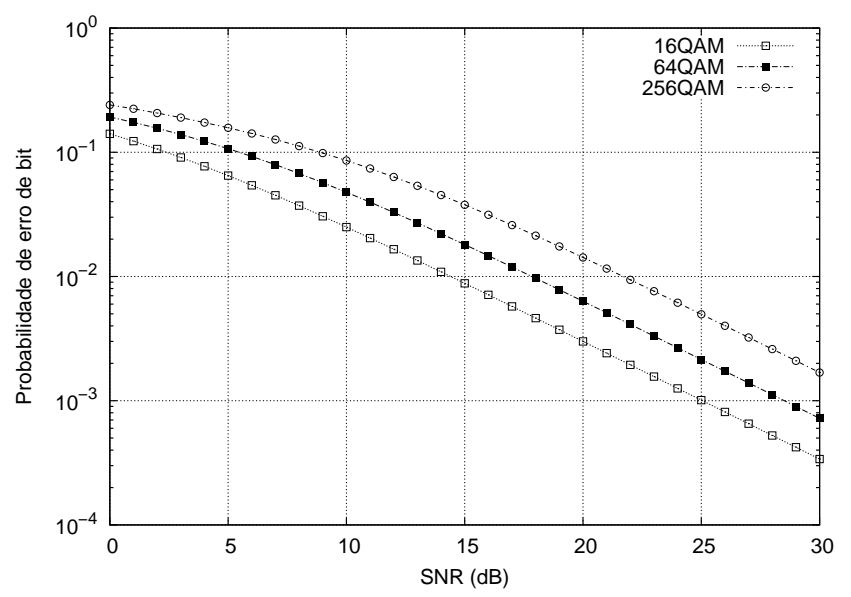

Fig. 4. Probabilidade de erro de bit de um sistema M-QAM sob desvanecimento Nakagami para $m=0.95$ e diferentes valores de $M$.



Fig. 5. Probabilidade de erro de bit de um sistema 256-QAM sob desvanecimento Nakagami para diferentes valores de $m, m>1$. 
susceptíveis ao desvanecimento, principalmente ao desvanecimento Rayleigh, comum em muitos ambientes urbanos. Apesar dessa susceptibilidade, os esquemas com grandes constelações são muito usados, principalmente quando são necessárias taxas de transmissão de bit mais elevadas.

O último conjunto de curvas, mostrado na Fig. 8, é referente à modulação M-PAM para $m=0.95$ e diferentes valores de $M$. O valor $m=0.95$ foi escolhido por estar entre 0.5 e 1.0 e ser próximo de 1.0 (caso em que a distribuição Nakagami se aproxima da Rayleigh). Curvas para $m>1.0$ podem ser obtidas por meio das Equações (29) e (32).

\section{CONCLUSÃO}

Neste trabalho foram obtidas expressões exatas para o cálculo da probabilidade de erro de bit dos esquemas de modulação M-QAM quadrado e M-PAM para desvanecimento Nakagami. As expressões obtidas em termos da função hipergeométrica englobam resultados obtidos para o desvanecimento Rayleigh e podem ser avaliadas numericamente por meio de pacotes matemáticos como Maple e Matemática. Adicionalmente, foi fornecida uma expressão para valores inteiros do parâmetro $m$ em termos apenas da Equação (23) em [7]. A partir dos resultados obtidos verifica-se que a probabilidade de erro aumenta à medida que o parâmetro $m$ da distribuição diminui. À medida que $m$ aumenta, a intensidade do desvanecimento diminui e tanto as constelações maiores quanto as menores sofrem menos erros de demodulação. Percebese também pelas curvas obtidas que a relação sinal-ruído do sistema precisa ser aumentada para que se possa transmitir mais bits extras por símbolo, com a mesma probabilidade de erro de bit.

\section{REFERÊNCIAS}

[1] J. Lu, K. B. Lefaief, J. C. Chuang and M. L. Liou. "M-PSK and M-QAM BER computation using signal-space concepts". IEEE Transactions on Communications, vol. 47, no. 2, pp. 181-184, 1999.

[2] L. L. Yang and L. Hanzo. "A recursive algorithm for error probability evaluation of M-QAM”. IEEE Communications Letters, vol. 4, no. 10, pp. 304-306, 2000.

[3] P. M. Fortune, L. Hanzo and R. Steele. "On the computation of 16QAM and 64-QAM performance in Rayleigh-fading channels". IEICE Transactions on Communications, vol. E75B, no. 6, pp. 466-475, 1992.

[4] M. G. Shayesteh and A. Aghamohammadi. "On the error probability of linearity of modulated signals on frequency-flat Ricean, Rayleigh and AWGN channels." IEEE Transactions on Communications, vol. 43, no. 2, pp. 1454-1466, 1995.

[5] J. G. Proakis. Digital Communications. McGraw-Hill, New York, 3 edition, 1995.

[6] K. Cho and D. Yoon. "On the general BER expression of one and two dimensional amplitude modulations". IEEE Transactions on Communications, vol. 50, no. 7, pp. 1074-1080, 2002.

[7] W. T. A. Lopes, F. Madeiro and M. S. Alencar'. "Um novo método para o cálculo da probabilidade de erro de bit de esquemas de modulação QAM sujeitos ao desvanecimento Rayleigh". Revista da Sociedade Brasileira de Telecomunicações, vol. 19, no. 2, pp. 58-67, Agosto 2004.

[8] M. S. Alencar. Telefonia Celular Digital. Ed. Érica Ltda, 2004.

[9] A. Papoulis. Probability, Random Variables and Stochastic Processes. McGraw-Hill, Inc., 1991.

[10] M. Abramowitz and I. A. Stegun. Handbook of Mathematical Functions. Dover pubications, Inc., New York, 1970.

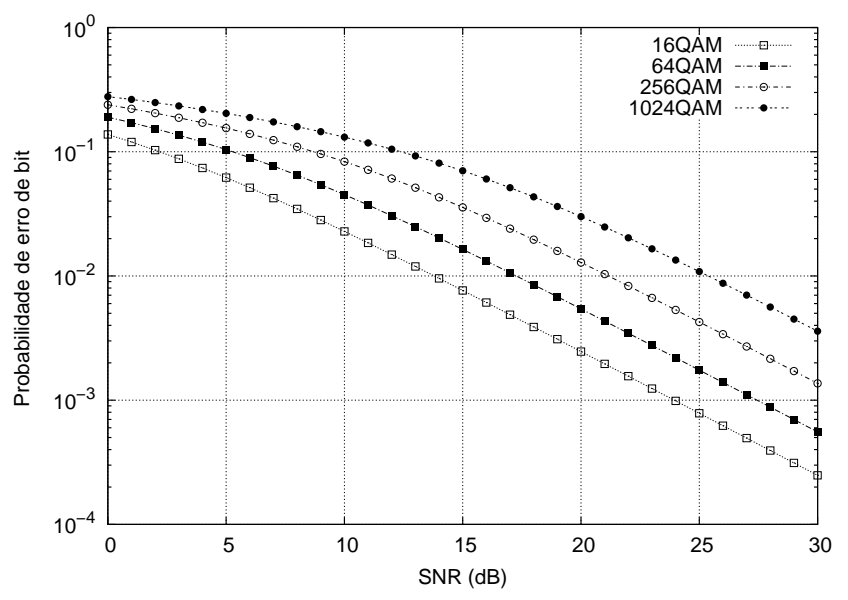

Fig. 6. Probabilidade de erro de bit de um sistema M-QAM sob desvanecimento Nakagami para $m=1$ e diferentes valores $M$.

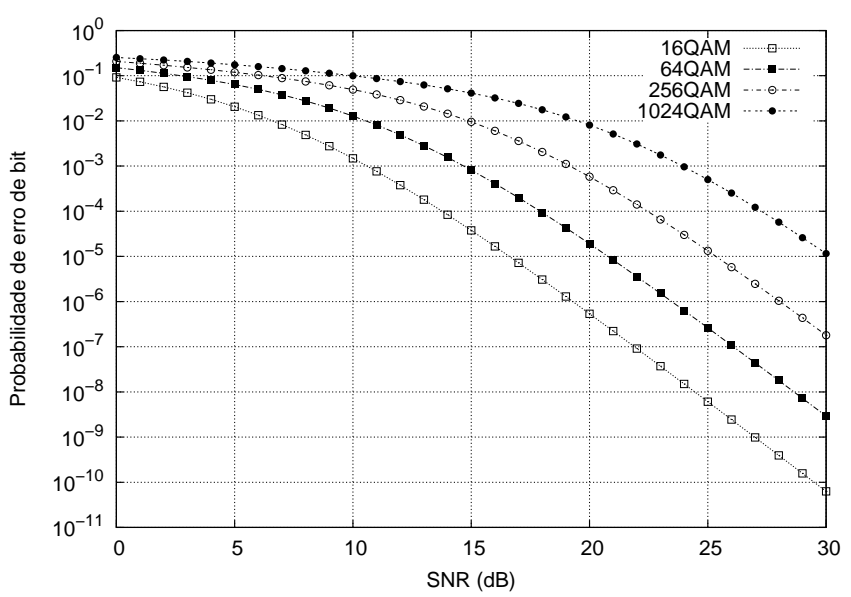

Fig. 7. Probabilidade de erro de bit de um sistema M-QAM sob desvanecimento Nakagami para $m=4$ e diferentes valores $M$.

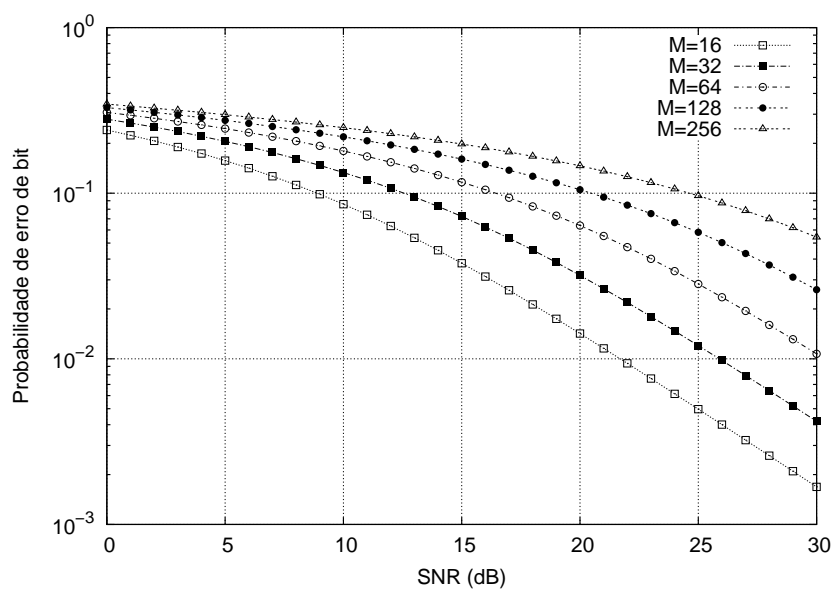

Fig. 8. Probabilidade de erro de bit de um sistema M-PAM sob desvanecimento Nakagami para $m=0.95$ e diferentes valores $M$. 